\title{
Phillyrin Relieves Lipopolysaccharide-Induced AKI by Protecting Against Glycocalyx Damage and Inhibiting Inflammatory Responses
}

\author{
Dong Zhang $@{ }^{1}$ Boyang Qi, ${ }^{1}$ Dongxiao Li, ${ }^{1}$ Jiali Feng, ${ }^{1}$ Xiao Huang, ${ }^{1}$ Xiaohong Ma, \\ Lina Huang, ${ }^{2}$ Xiaozhi Wang, ${ }^{1,3}$ and Xiangyong Liu ${ }^{2,3}$
}

\begin{abstract}
Damage to the integrity of heparin sulfate (HS) in the endothelial glycocalyx is an important factor of glomerular filtration barrier dysfunction, which is the basic pathological feature of acute kidney injury (AKI). AKI is a common clinical critical illness with few drugs options offering effective treatment. Phillyrin (Phil), the main pharmacological component of Forsythia suspensa, possesses a wide range of pharmacological activities. However, the effects of Phil on lipopolysaccharide (LPS)-induced AKI have yet to be reported. The aim of the present study is to analyze the effects of Phil on HS damage and inflammatory signaling pathways in LPS-induced AKI. Results revealed that Phil reduces pathological changes and improves renal function in LPS-induced AKI. Further analysis indicated that Phil effectively protects against glycocalyx HS degradation in LPS-stimulated EA.hy926 cells in vitro and LPS-induced AKI mice in vivo. The protective effect of Phil on HS damage may be associated with the isolate's ability to suppress the production of reactive oxygen species, and decrease expression levels of cathepsin $\mathrm{L}$ and heparanase in vitro and in vivo. In addition, ELISA and Western blot results revealed that Phil inhibits the activation of the NF$\mathrm{KB}$ and MAPK signaling pathways and decreases the levels of inflammatory cytokines (IL$1 \beta$, IL-6, and TNF- $\alpha$ ) in LPS-induced ARDS mice. In general, protection against endothelial glycocalyx HS damage and inhibition of inflammatory responses by Phil may be used as treatment targets for LPS-induced AKI.
\end{abstract}

KEY WORDS: acute kidney injury; heparin sulfate; phillyrin; inflammatory signaling pathways.

Dong Zhang, Boyang Qi and Dongxiao Li contributed equally to this work.

\footnotetext{
${ }^{1}$ Department of Respirator Medicine and Intensive Care Unit, Affiliated Hospital of Binzhou Medical University, Binzhou, China

${ }^{2}$ Department of Cell Biology, Binzhou Medical University, Yantai, China

${ }^{3}$ To whom correspondence should be addressed to Xiaozhi Wang at Department of Respirator Medicine and Intensive Care Unit, Affiliated Hospital of Binzhou Medical University, Binzhou, China. E-mail: hxicuwxz@163.com; and Xiangyong Liu at Department of Cell Biology, Binzhou Medical University, Yantai, China. E-mail: liuxiangyong81@gmail.com
}

\section{INTRODUCTION}

Acute kidney injury (AKI), one of the most common critical illnesses, is mainly characterized by short-term renal function decline [1]. The etiology of AKI is complex and includes sepsis, cardiovascular diseases, and urinary tract obstruction [2-4]. At present, no effective treatment is yet available for AKI. Lipopolysaccharide (LPS) is a major component of the extracellular wall of Gram-negative 
bacteria and the most important pathogenic factor for AKI. LPS is widely used to stimulate animal models of inducing AKI $[5,6]$.

Endothelial glycocalyx damage has an important impact on the pathogenesis of renal injury. The glycocalyx covers the surface of the vascular endothelial cells to regulate the glomerular filtration barrier function. Proteoglycans, such as heparin sulfate (HS), are the main component and form the skeletal of the glycocalyx. Glycocalyx HS is susceptible to degradation by a number of enzymes and oxidative damage. Marjolein Ggarsen et al. [7] reported that heparanase (HPA) degrades glycocalyx HS in the diabetic wild type mice. Henry Puerta-Guardo et al. [8] reported that cathepsin L is associated with dengue virus NS1-induced the endothelial glycocalyx damage. Excessive oxidation produces a large amount of reactive oxygen species (ROS), which induce oxidative damage in kidney tissues and the endothelial glycocalyx $[9,10]$. Activation of inflammatory signaling pathways (e.g., NF-кB and MAPK) can also induce excessive inflammatory cytokines (e.g., TNF- $\alpha$, IL6 , and IL-1 $\beta$ ) and affect renal vascular cell function, thereby leading to kidney damage. However, the mechanism of glycocalyx HS damage in LPS-induced AKI is unknown.

Forsythia suspensa is a well-known traditional Chinese medicine, listed in the Chinese pharmacopeia as more than 40 Chinese herbal preparations containing Forsythia suspensa [11]. Phillyrin (Phil) is an active lignan compound, which mainly exists in the bark and fruit of Forsythia suspensa, and is the main pharmacological component of Forsythia suspensa [11]. Phil exerts many pharmacological effects, such as anti-inflammatory [12], antioxidant [13], antiobesity [14], and antiviral [15] activities. To date, the therapeutic effects of Phil on AKI have not been reported yet.

In the present study, the effects and possible mechanisms of Phil in LPS-induced AKI in vitro and in vivo were investigated. The results may be provided a new theoretical basis for the development of therapeutic drugs for AKI.

\section{MATERIALS AND METHODS}

\section{Materials}

Phil (purity $>99.5 \%$ ) was acquired from Chengdu Must Bio-Technology, China. LPS was obtained from Escherichia coli O55:B5 (Sigma-Aldrich, China). HRPconjugated goat anti-rabbit $\operatorname{IgG}$ and $\beta$-actin were provided by Beijing Zhongshan Golden Bridge Biological Technology, China. Blood urea nitrogen (BUN) and serum creatinine ( $\mathrm{SCr}$ ) assay kits were supplied by Nanjing Jiancheng
Bioengineering Institute, China. $\mathrm{N}$-acetyl cysteine (NAC, a ROS scavenger) and ELISA determination kits of ROS, TNF- $\alpha$, IL- 6 , and IL- $1 \beta$ were purchased from Beyotime Biotechnology, China. Rabbit monoclonal antibodies to ERK, JNK, p38, p-ERK, p-JNK, p-p38, NF-kB p65, $\mathrm{I} \kappa \mathrm{B} \alpha, \mathrm{p}-\mathrm{I} \kappa \mathrm{B} \alpha$, Lamin B1, HPA, and mouse albumin ELISA kit were purchased from Abcam Trading Company, USA. Mouse HS ELISA kit was purchased from Mlbio, China. Mouse polyclonal antibody to HS was purchased from AMS Biotechnology (Europe). Cathepsin L (CTL) polyclonal antibody and cathepsin L inhibitor (CTL inhib) were purchased from Santa Cruz Biotechnology. Fluorescein isothiocyanate (FITC)-conjugated anti-goat IgG was purchased from Zhongshan Golden Bridge Biotechnology (Beijing, China).

\section{Cell Culture and Treatment}

EA.hy926 cells were derived from the fusion of human umbilical vein endothelial cells with a thioguanineresistant clone of A549 by exposure to polyethylene glycol [16]. EA.hy926 cells were purchased from China Cell Line Bank (Beijing, China). EA.hy926 cells were grown to confluence and cultured in DMEM supplemented with $10 \%$ heated-inactivated fetal bovine serum (Invitrogen/ Gibco Life Technologies, Carlsbad, CA), penicillin (100 U/mL), and streptomycin $(100 \mathrm{~g} / \mathrm{mL})$ in an incubator with a humidified atmosphere of $95 \%$ air and $5 \% \mathrm{CO}_{2}$ at $37{ }^{\circ} \mathrm{C}$. Cells at $80-90 \%$ confluence were used for all assays. In a preliminary experiment, MTT assay results showed that $20 \mu \mathrm{M}$ Phil presents no cytotoxicity against EA.hy926 cells; however, concentrations greater than $20 \mu \mathrm{M}$ induced cytotoxicity. Therefore, $20 \mu \mathrm{M}$ was selected as the Phil with pretreatment concentration.

To analyze the effect of ROS on glycocalyx HS damage in EA.hy926 cells, EA.hy926 cells were sorted into control, NAC, Phil, LPS, Phil + LPS, and NAC + LPS groups. Cells in the control group were cultured in the culture medium without intervention. Cells in the NAC group were cultured in the culture medium with $1 \mathrm{mM}$ NAC for $1 \mathrm{~h}$. Cells in the Phil group were cultured in the culture medium with $20 \mu \mathrm{M}$ Phil for $1 \mathrm{~h}$. Cells in the LPS group were cultured in the culture medium with LPS $(1 \mu \mathrm{g} / \mathrm{mL})$ for $12 \mathrm{~h}$. Cells in the Phil + LPS group were pretreated with $20 \mu \mathrm{M}$ Phil for $1 \mathrm{~h}$, washed three times with phosphate buffered solution (PBS), and stimulated with LPS $(1 \mu \mathrm{g} / \mathrm{mL})$ for $12 \mathrm{~h}$. Finally, cells in the $\mathrm{NAC}+$ LPS group were pretreated with $1 \mathrm{mM}$ NAC for $1 \mathrm{~h}$, washed three times with PBS, and then stimulated with LPS $(1 \mu \mathrm{g} / \mathrm{mL})$ for $12 \mathrm{~h}$. 
To analyze the effect of enzymes on glycocalyx HS damage in EA.hy926 cells, EA.hy926 cells were sorted into the control, CTL inhib (cathepsin L inhibitor), Phil, LPS, Phil + LPS, and CTL inhib + LPS groups. Cells in the control group were cultured in the culture medium without intervention. Cells in the CTL inhib group were cultured in the culture medium with cathepsin L inhibitor $(10 \mu \mathrm{M})$ for $1 \mathrm{~h}$. Cells in the Phil group were cultured in the culture medium with $20 \mu \mathrm{M}$ Phil for $1 \mathrm{~h}$. Cells in the LPS group were cultured in the culture medium with LPS $(1 \mu \mathrm{g} / \mathrm{mL})$ for $12 \mathrm{~h}$. Cells in the Phil + LPS group were pretreated with $20 \mu \mathrm{M}$ Phil for $1 \mathrm{~h}$, washed three times with PBS, and then stimulated with LPS $(1 \mu \mathrm{g} / \mathrm{mL})$ for $12 \mathrm{~h}$. Finally, cells in the CTL inhib + LPS group were pretreated with cathepsin $\mathrm{L}$ inhibitor $(10 \mu \mathrm{M})$ for $1 \mathrm{~h}$, washed three times with PBS, and then stimulated with LPS $(1 \mu \mathrm{g} / \mathrm{mL})$ for $12 \mathrm{~h}$.

\section{AKI Modeling and Grouping of Mice}

C57BL/6 male mice aged 8-10 weeks and weighing 18-20 g were purchased from Jinan Pengyue Experimental Animal Breeding Co., Ltd. (Shandong). Mice were housed in an environment at $21 \pm 1{ }^{\circ} \mathrm{C}$ under $12 \mathrm{~h} / 12 \mathrm{~h}$ light/darknes and $70-80 \%$ humidity. The animal care and experimental procedures were conducted in accordance with the Guide for the Care and Use of Laboratory Animals, and the protocol of this study was approved by the Institutional Animal Care and Use Committee of Binzhou Medical University Hospital. During Phil pretreatment, mice were randomly divided into four groups ( $n=8$ in each group): control, Phil, LPS, and Phil + LPS. The dosage and mode of Phil administration were derived from the pre-experiment and previous reports
[12]. Mice in the Phil and Phil + LPS groups were intragastrically administered with Phil at dosage of $40 \mathrm{mg} / \mathrm{kg}$ once a day for 7 days [17]. Mice in the control and LPS groups were intragastrically administered with an equal volume of normal saline. After 7 days of continuous gavage, mice in the LPS and Phil + LPS groups were intraperitoneally injected with $10 \mathrm{mg} / \mathrm{kg}$ LPS to induce AKI [6] (Fig. 1). Mice in the control and Phil groups were injected with an equal volume of normal saline to the peritoneal cavity. After LPS stimulation for $12 \mathrm{~h}$, all mice were subjected to anesthesia with $4 \%$ chloral hydrate prior to obtaining kidney tissue, urine, and blood samples.

\section{Pathological Changes in Kidney Tissues}

Kidney tissues were fixed with $4 \%$ paraformaldehyde for 4 days and then subjected to alcohol gradient dehydration and paraffin immersion and embedding. Paraffin-embedded renal tissues were cut into 5- $\mu \mathrm{m}$ sections for hematoxylin and eosin (HE) staining. Pathological changes in the kidney tissues, such as destruction or dilation of tubular structures and loss of the brush boarder, were observed under an optical microscope (Olympus Optical, Japan).

\section{Detection of SCr, BUN, and Albumin Levels}

Renal function status was evaluated by detecting changes in serum SCr and BUN and urinary albumin levels $[18,19]$.

Blood samples. The head of the mouse was fixed, the blood collector was placed at an angle of $45^{\circ}$ relative to the face of the mouse, the needle was inserted between the

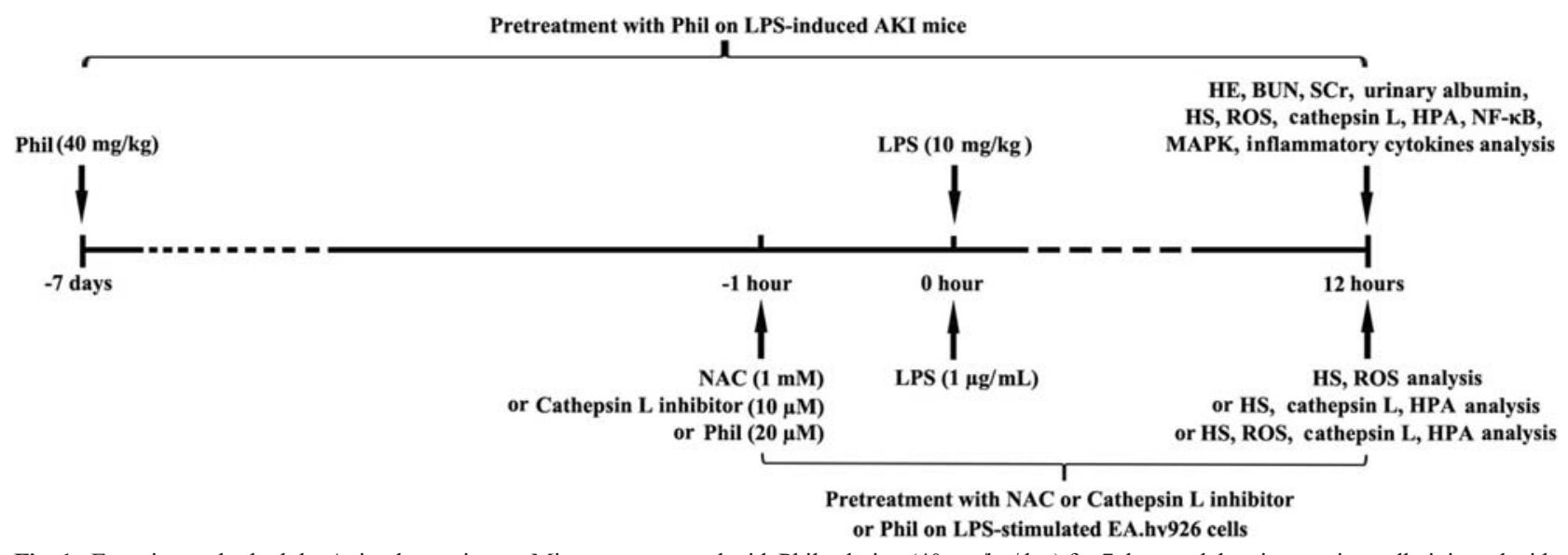

Fig. 1. Experimental schedule. Animal experiment: Mice were gavaged with Phil solution ( $40 \mathrm{mg} / \mathrm{kg} / \mathrm{day})$ for 7 days and then intraperitoneally injected with $10 \mathrm{mg} / \mathrm{kg}$ LPS to induce AKI. Cell experiment: EA.hy926 cells were pretreated with $1 \mathrm{mM} \mathrm{NAC}$ or $10 \mu \mathrm{M}$ cathepsin L inhibitor or $20 \mu \mathrm{M}$ Phil for $1 \mathrm{~h}$ and then stimulated by $1 \mu \mathrm{g} / \mathrm{mL}$ LPS for $12 \mathrm{~h}$. 
lower eyelid and eyeball, and blood was gently moved to the direction of the fundus. Blood was collected by rotating the blood vessel to maintain a horizontal position by cutting the venous plexus, and the blood could be taken out with slight attraction. Samples were allowed to stand for $30 \mathrm{~min}$ and then centrifuged at $4000 \mathrm{rpm}$ for $20 \mathrm{~min}$ at $4{ }^{\circ} \mathrm{C}$. The supernatant was collected and used to determine $\mathrm{SCr}$ and BUN levels according to the instructions of the corresponding ELISA kits. SCr and BUN contents were expressed as milligrams per liter ( $\mathrm{mg} / \mathrm{L})$.

Urine samples. Mice were placed in individual metabolic cages and provided drinking water but no food. Urine collected from the mice was centrifuged within $24 \mathrm{~h}$ and centrifuged at $800 \mathrm{~g}$ for $10 \mathrm{~min}$ at $4{ }^{\circ} \mathrm{C}$. The supernatant was collected and used to determine albumin contents according to the instructions of the mouse urinary albumin kit. Urinary albumin content was expressed as nanograms per milliliter (ng/mL).

\section{Determination of HS, HPA, and Cathepsin L in Vitro and in Vivo}

To detect HS, HPA, and cathepsin L levels in EA.hy926 cells, the cells were fixed with $4 \%$ paraformaldehyde, washed with PBS, and treated with serum blockers. Then, the cells were incubated with antibodies (HS, HPA, or cathepsin L) and subjected to fluorescence secondary antibody and DAPI staining. Images were acquired with a fluorescence microscope (Olympus BX53). Relative quantitative data were obtained by using Image $\mathrm{J}$, and all results were statistically analyzed by using SPSS 17.0.

To detect HS, HPA, and cathepsin L levels in the glomerulus of AKI animals, 5- $\mu \mathrm{m}$ tissue sections were deparaffinized, hydrated, subjected to antigen retrieval, and permeabilized with $0.5 \%$ Triton $\mathrm{X}-100$. The sections were washed with PBS, incubated with goat serum, and then treated with primary antibodies (HS, HPA, or cathepsin L) overnight at $4{ }^{\circ} \mathrm{C}$. Thereafter, the sections were washed with PBS and incubated with FITC-conjugated secondary antibodies for $30 \mathrm{~min}$ at room temperature. Finally, the cell nuclei were stained with DAPI. Images were obtained by using a fluorescence microscope (Olympus BX53, Japan). Relative quantitative data were obtained by using Image $J$, and all results were statistically analyzed by using SPSS 17.0.

\section{Detection of ROS Contents in Vitro and in Vivo}

ROS contents in vitro and in vivo were detected by the $2^{\prime}, 7^{\prime}$-dichlorodihydrofluorescein diacetate (DCFH-DA) method. After entering the cell, DCFHDA is hydrolyzed into DCFH, which can be oxidized by ROS to generate fluorescent 2', 7'dichlorofluorescein (DCF) [20].

After pretreatment, EA.hy926 cells were incubated in the culture medium containing $10 \mu \mathrm{M} \mathrm{DCFH}-$ DA for 20 min and washed with PBS. DCF fluorescence was determined by fluorescence microscopy (Olympus BX53, Japan). Relative quantitative data were obtained by using Image $J$, and all results were statistically analyzed by using SPSS 17.0.

Mice were subjected to anesthesia with $4 \%$ chloral hydrate prior to obtaining kidneys. The kidneys were placed in a precooled PBS homogenate (kidney:PBS, $1 \mathrm{mg}: 5 \mu \mathrm{L}$ ). Approximately $190 \mu \mathrm{L}$ of the homogenate and $10 \mu \mathrm{L}$ of DCFH-DA (final concentration, $1 \mathrm{mM}$ ) were added to a 96-well plate and incubated at $37{ }^{\circ} \mathrm{C}$ for $30 \mathrm{~min}$. The fluorescence intensity of DCF was measured at an excitation wavelength of $485 \mathrm{~nm}$ and emission wavelength of $530 \mathrm{~nm}$. The content of ROS in kidneys was expressed as micromoles $(\mu \mathrm{M})$ of DCF formed per milligram $(\mathrm{mg})$ of protein.

\section{Detection of Inflammatory Cytokines and Shedding of HS in Serum}

Blood samples were collected from the eyeballs of mice, allowed to stand for $30 \mathrm{~min}$, and centrifuged at $4000 \mathrm{rpm}$ for $20 \mathrm{~min}$ at $4{ }^{\circ} \mathrm{C}$. The supernatant was collected and used to determine IL- 6 , TNF- $\alpha$, IL- $1 \beta$, and HS levels according to the instructions of the corresponding ELISA kits. IL- 6 , TNF- $\alpha$, and IL- $1 \beta$ levels were expressed as picograms per milliliter (pg/ $\mathrm{mL}$ ). HS level was expressed as nanograms per milliliter $(\mathrm{ng} / \mathrm{mL})$.

\section{Western Blot Analysis}

After LPS stimulation for $12 \mathrm{~h}$, mice were anesthetized with $4 \%$ chloral hydrate. Kidneys were obtained and stored in a refrigerator at $-80^{\circ} \mathrm{C}$. Protein in kidneys was extracted according to the instructions of a protein extraction kit (Beyotime, China), and protein concentrations were measured with a BCA protein assay kit (Beyotime).

The protein was separated by SDS-PAGE and transferred onto polyvinylidene fluoride (PVDF) membranes. 
The membranes were incubated with 5\% milk for $2 \mathrm{~h}$ at room temperature, added with primary antibodies (nonphosphorylated forms of NF-kB p65, IkB $\alpha$, ERK, JNK, and p38; phosphorylated forms of IKB $\alpha$, ERK, $\mathrm{JNK}$, and $\mathrm{p} 38$ ), and placed in a refrigerator overnight at $4{ }^{\circ} \mathrm{C}$. The PVDF membranes were then washed with TBST and incubated with the secondary antibody for $2 \mathrm{~h}$ at room temperature. Finally, the PVDF membranes were added with developer solution and photographed. Protein ratios were normalized against $\beta$-actin or lamin $\mathrm{B} 1$, and protein bands were quantitatively analyzed by using Image $\mathrm{J}$.

\section{Statistical Analysis}

All data were expressed as mean \pm standard deviation. Differences among groups were analyzed by Student's $t$ test, one-way ANOVA, and SNK test. Differences at $P<0.05$ was considered statistically significant. All statistical analysis was performed by SPSS 17.0 (Statistical product and service solutions 17.0, IBM Corp).

\section{RESULTS}

\section{Effects of Phil on LPS-Induced Kidney Histopathologic Changes}

The effect of Phil on kidney pathological changes in the kidney of LPS-induced AKI mice was evaluated by HE staining. Compared with those of the control group, changes in the Phil group were insignificant (Fig. 2a, b). Mice in the LPS group presented significant renal damage, such as destroyed or dilated tubular structures, degenerated vacuoles, and lost brush borders (Fig. 2a, b). These pathological changes improved significantly in LPS-induced AKI mice pretreated with Phil.

\section{Effects of Phil on Kidney Function in LPS-Induced AKI Mice}

The kidney function status of mice may be reflected by serum BUN and $\mathrm{SCr}$ and urinary albumin levels $[18$, 19]. The SCr, BUN, and albumin levels significantly

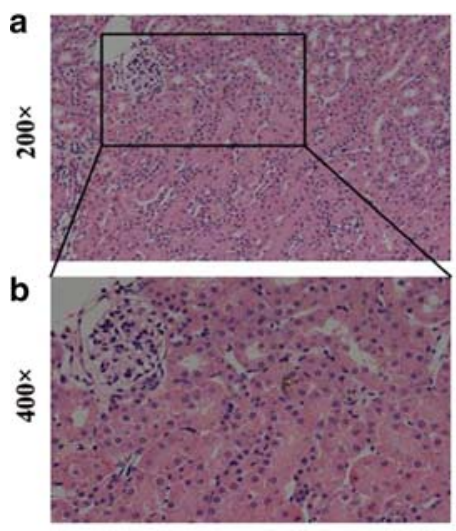

Control

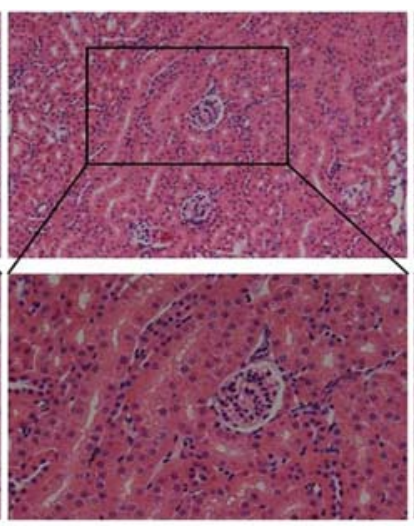

Phil

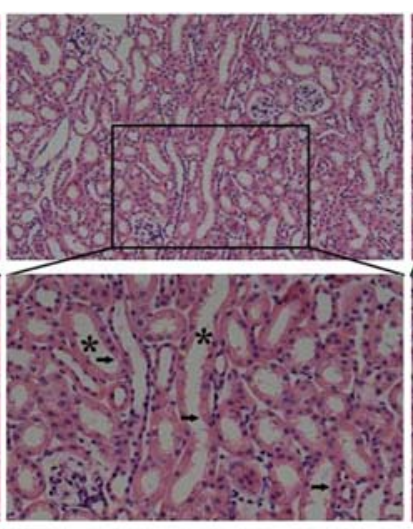

LPS

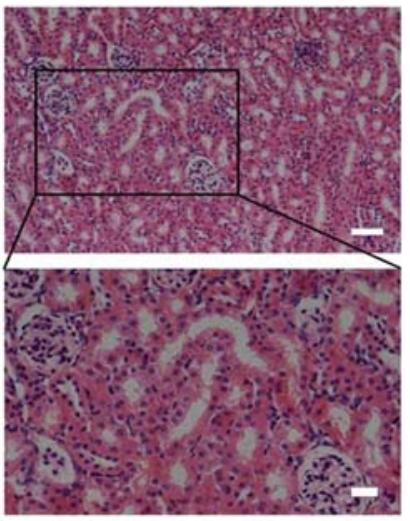

Phil+LPS
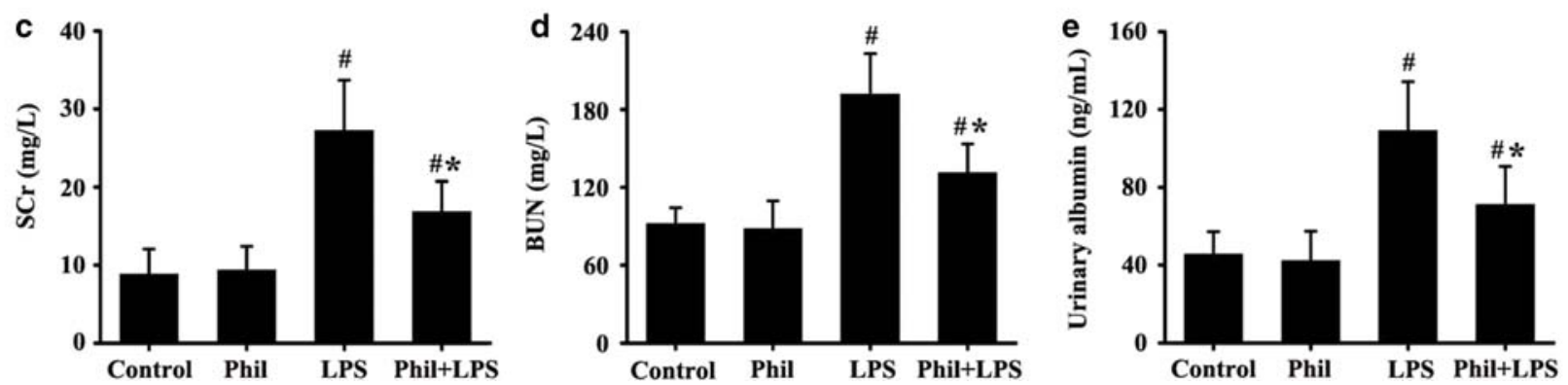

Fig. 2. Effects of Phil on the histopathological changes and renal function of LPS-induced AKI mice. Histological evaluation of the kidney was conducted by HE staining. a Magnification, $\times 200$; scale bar, $50 \mu \mathrm{m}$. b Magnification, $\times 400$; scale bar, $20 \mu \mathrm{m}$. Serum c SCr and d BUN and e urinary albumin levels were detected by using the corresponding ELISA kits. All data are presented as the mean $\pm \mathrm{SD}$ of three independent experiments $\left(n=8\right.$ in each group). ${ }^{\#} P<0.05$ compared with the control group; ${ }^{\# * P}<0.05$ compared with the LPS group. 


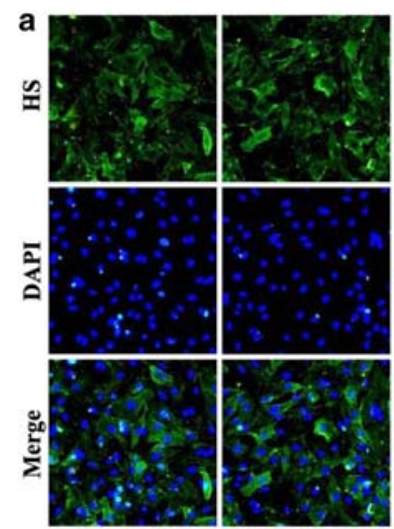

Control

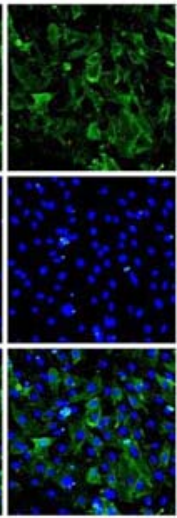

Phil

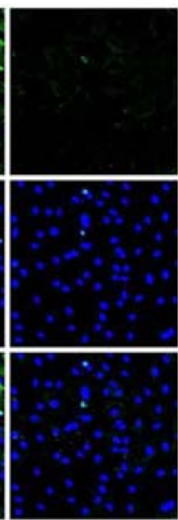

LPS

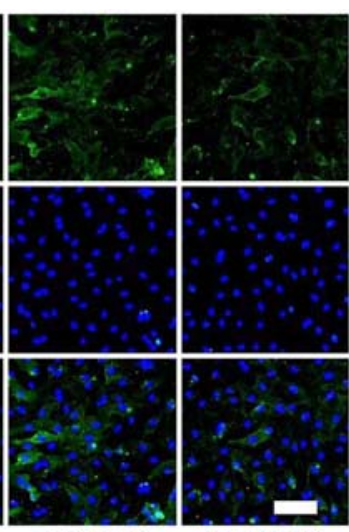

Phil+LPS

NAC + LPS
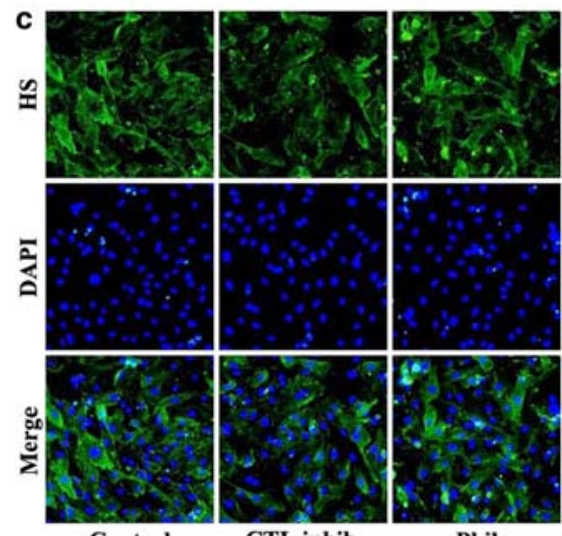

Phil

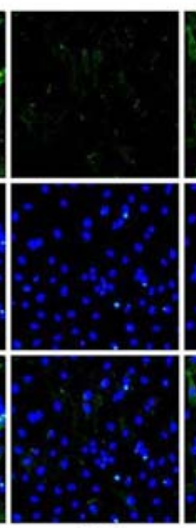

LPS
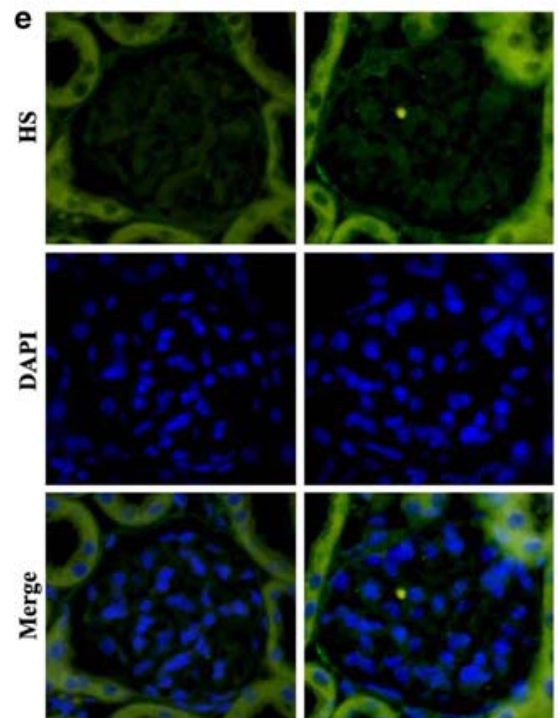

Control

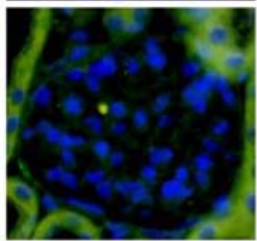

Phil
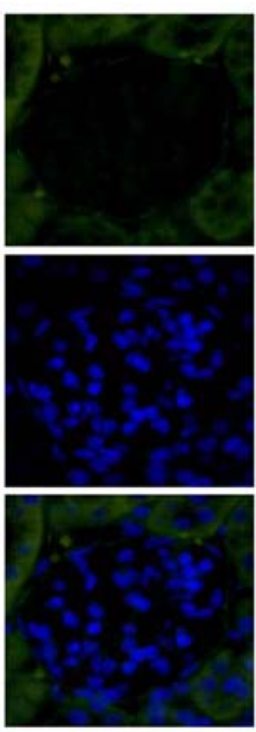

LPS
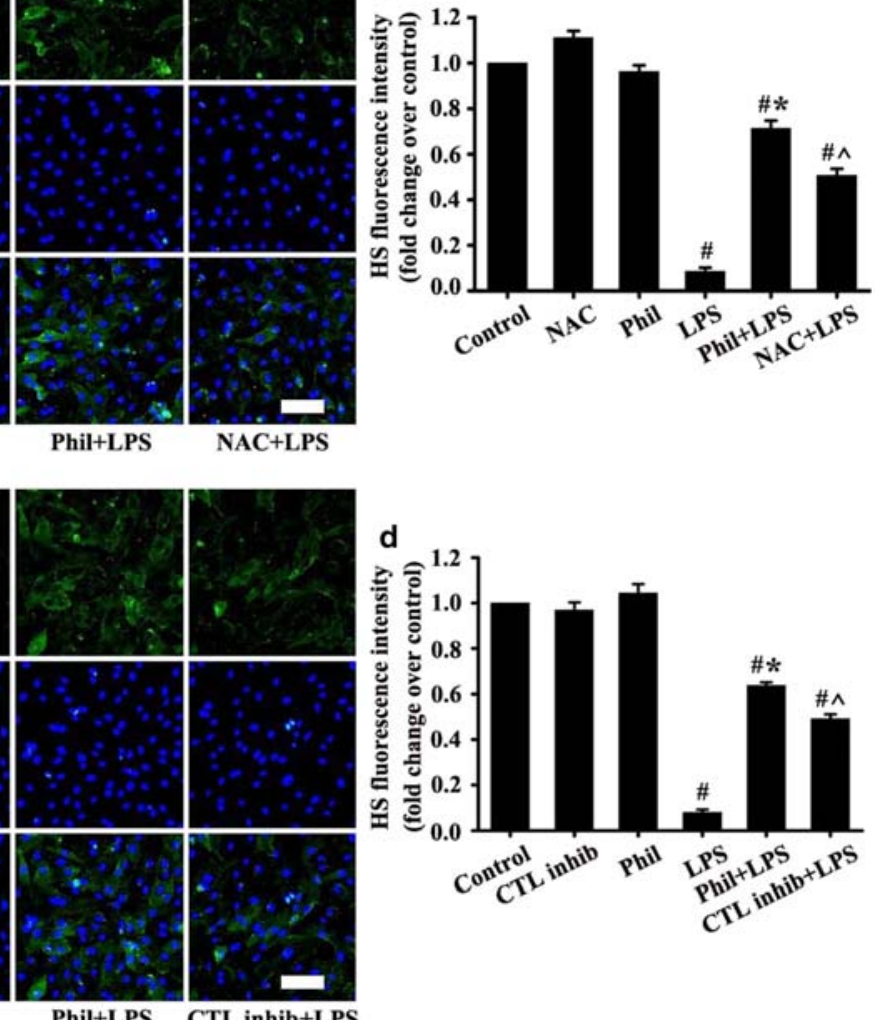

CTL inhib+LPS
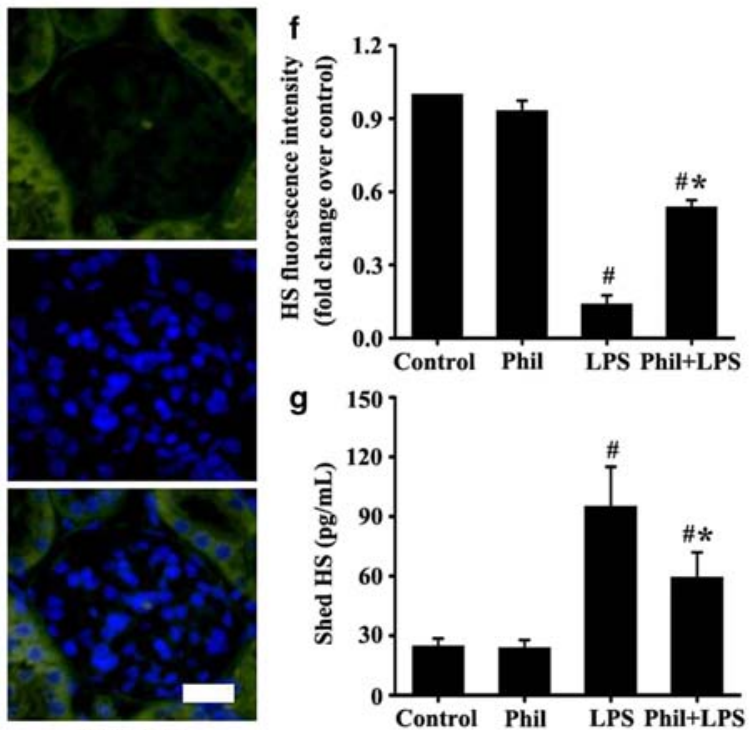

Fig. 3. Effects of Phil on HS damage in LPS-stimulated EA.hy926 cells and mice. HS contents (a, c Magnification, $\times 100$; scale bar, $100 \mu$ m; e Magnification, $\times 400$; scale bar, $20 \mu \mathrm{m}$ ) were detected by immunofluorescence. b, d, f Fluorescence intensity analysis of a, c, and e, respectively. $\mathbf{g}$ Shedding of HS in serum was detected by the corresponding ELISA kit. All data are presented as the mean \pm SD of three independent experiments $(n=8$ in each group). ${ }^{\#} P<0.05$ compared with the control group; ${ }^{\# *} P<0.05$ compared with the LPS group; ${ }^{\# \wedge} P<0.05$ compared with the LPS group. 
increased in LPS-induced AKI mice (Fig. 2c-e). This finding indicates impairments in kidney function, consistent with previous reports. However, SCr, BUN, and albumin levels significantly decreased after Phil pretreatment (Fig. 2c-e). Thus, pretreatment with Phil can improve the kidney function of LPS-induced AKI mice.

\section{Effects of Phil on LPS-Induced Glycocalyx HS Degra- dation In Vitro and In Vivo}

HS is the main component of the endothelial glycocalyx and could be used to evaluate glycocalyx damage. The results showed that HS is evidently exfoliated after LPS stimulation in vitro and in vivo (Fig. 3a-g). However, glycocalyx HS shedding decreased in the NAC + LPS and CTL inhib + LPS groups (Fig. 3a-d). Pretreatment with Phil significantly inhibited the shedding of HS in vitro and in vivo (Fig. 3a-g). These results indicate that the mechanisms of glycocalyx HS damage are closely related to the expression levels of cathepsin L and HPA and the production of ROS in EA.hy926 cells and LPS-induced AKI mice. Phil may decrease LPS-induced HS shedding by reducing ROS production and inhibiting the expression levels of cathepsin L and HPA in EA.hy926 cells and mice.

\section{Effects of Phil on LPS-Induced ROS Production In Vitro and In Vivo}

Oxidative damage to renal tissues and the endothelial glycocalyx can occur during the pathological process of several diseases. Oxidative damage was evaluated by measuring ROS levels in vitro and in vivo. Compared with those of the control group, the ROS levels of LPS-stimulated EA.hy926 cells and mice significantly increased (Fig. 4ac). ROS levels significantly decreased after pretreatment with NAC or Phil (Fig. 4a-c). These results show that Phil can decrease ROS production to protect LPS-stimulated EA.hy926 cells and mice against oxidative damage.

\section{Effects of Phil on LPS-Induced Cathepsin L Expression In Vitro and In Vivo}

Cathepsin L expression levels increased after LPS stimulation in vitro and in vivo (Fig. 5a-d). However, cathepsin L expression levels in the CTL inhib + LPS and Phil + LPS groups significantly decreased (Fig. 5a-d). These results show that Phil can inhibit cathepsin L expression in vitro and in vivo.

\section{Effects of Phil on LPS-Induced HPA Expression In Vitro and In Vivo}

HPA is a specific degradation enzyme of glycocalyx HS shedding. Results showed that HPA expression increases after LPS stimulation in vitro and in vivo (Fig. 6a-d). However, HPA expression levels in the CTL inhib + LPS and Phil + LPS groups significantly decreased (Fig. 6a-d). These results show that pretreatment with cathepsin L inhibitor effectively inhibits HPA expression in EA.hy926 cells stimulated with LPS. Therefore, Phil may regulate HPA expression by inhibiting cathepsin $\mathrm{L}$ in LPS-stimulated EA.hy926 cells and mice.

\section{Effects of Phil on LPS-Induced Inflammatory Re- sponses in LPS-Induced AKI Mice}

AKI pathogenesis is accompanied by the production of inflammatory cytokines [21]. Compared with those in the control and Phil groups, the levels of inflammatory cytokines in groups with LPS-induced AKI significantly increased (Fig. 7a-c). However, pretreatment with Phil significantly decreased serum levels of inflammatory cytokines (Fig. 7a-c).

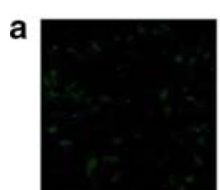

Control

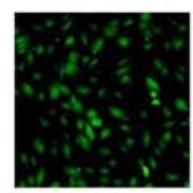

LPS

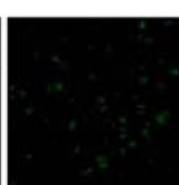

NAC

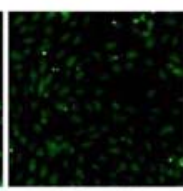

Phil+LPS

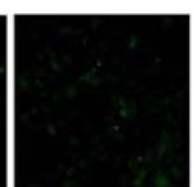

Phil

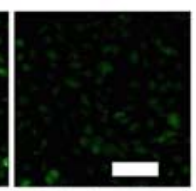

NAC+LPS
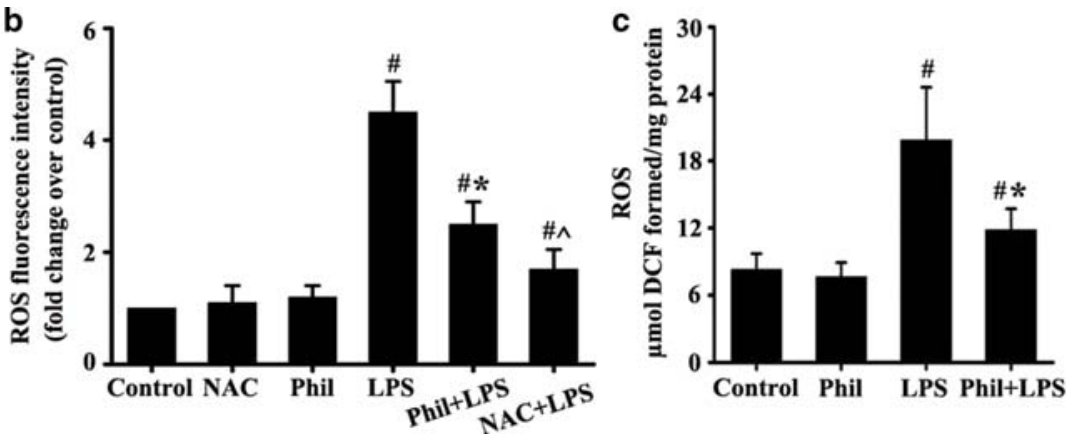

Fig. 4. Effects of Phil on ROS in LPS-stimulated EA.hy926 cells and mice. ROS levels in a EA.hy926 cells and $\mathbf{c}$ AKI mice were measured (magnification, $\times 100$; scale bar, $100 \mu \mathrm{m})$. b Fluorescence intensity analysis of $\mathbf{a}$. All data are presented as the mean \pm SD of three independent experiments $(n=8$ in each group). ${ }^{\#} P<0.05$ compared with the control group; ${ }^{\# * P}<0.05$ compared with the LPS group; ${ }^{\#} \wedge P<0.05$ compared with the LPS group. 

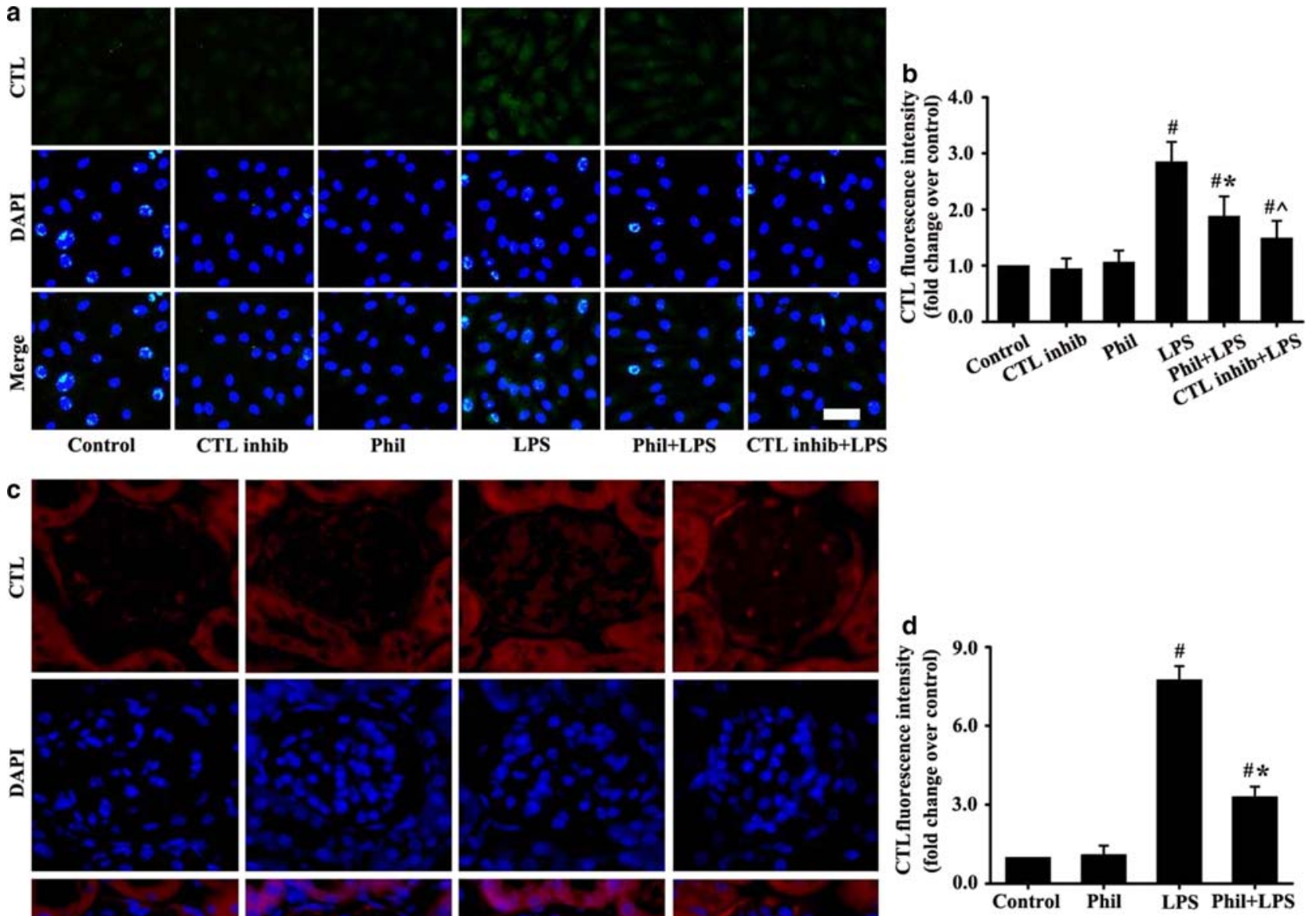

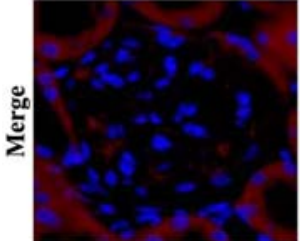

Control

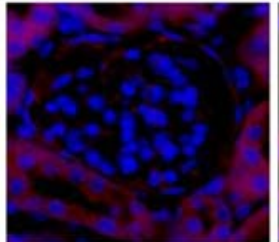

Phil

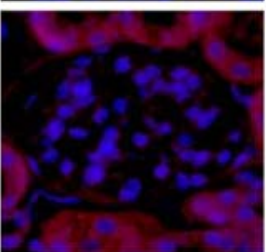

LPS

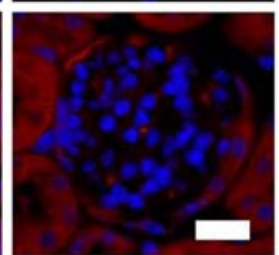

Phil+LPS

Fig. 5. Effects of Phil on cathepsin L (CTL) in LPS-stimulated EA.hy926 cells and mice. CTL contents a in vitro (magnification, $\times 200$; scale bar, $50 \mu \mathrm{m})$ and $\mathbf{c}$ in vivo (magnification, $\times 400$; scale bar, $20 \mu \mathrm{m}$ ) were detected by immunofluorescence. b, $\mathbf{d}$ Fluorescence intensity analysis of a and $\mathbf{c}$, respectively. All data are presented as the mean $\pm \mathrm{SD}$ of three independent experiments $\left(n=8\right.$ in each group). ${ }^{\#} P<0.05$ compared with the control group; ${ }^{*} P<0.05$ compared with the LPS group; ${ }^{\#} \wedge P<0.05$ compared with the LPS group.

Considering that the production of inflammatory cytokines is affected by the NF- $\mathrm{kB}$ and MAPK inflammatory pathways [12], we examined the effects of Phil on NF- $\mathrm{KB}$ and MAPK pathway activation. NF- $\mathrm{KB}$ p65 nuclear transfer and I $\mathrm{KB} \alpha$ phosphorylation were enhanced in LPS-induced AKI (Fig. 7d-i), thereby indicating activation of NF- $\mathrm{KB}$ signaling. Pretreatment with Phil significantly reduced the NF$\kappa \mathrm{B}$ p65 nuclear transfer and $\mathrm{I} \kappa \mathrm{B} \alpha$ phosphorylation induced by LPS (Fig. 7d-i). Moreover, phosphorylation levels of p38, ERK, and JNK significantly increased in LPS-induced AKI (Fig. 7j-o), thereby indicating activation of MAPK signaling. Pretreatment with Phil also significantly reduced the LPSinduced phosphorylation levels of p38, ERK, and JNK (Figs. 7j-o). Thus, Phil can inhibit the activation of the NF-KB and MAPK signaling pathways in LPS-induced AKI mice. 


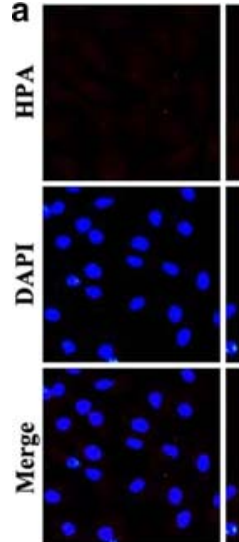

Control

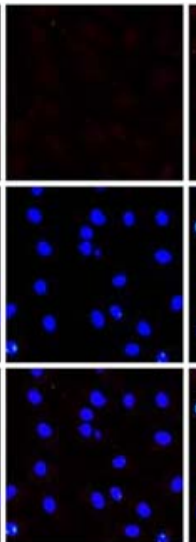

CTL inhib

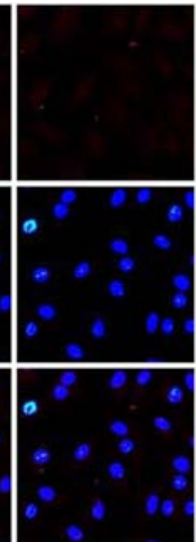

Phil

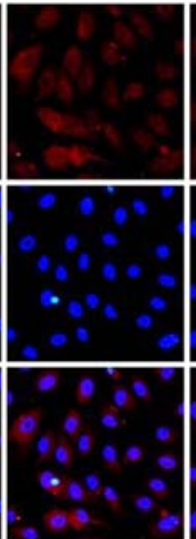

LPS

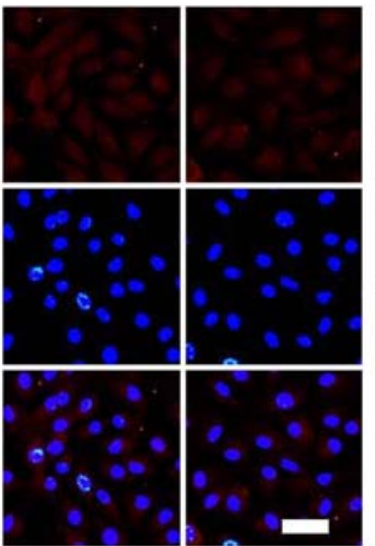

Phil+LPS CTL inhib+LPS b

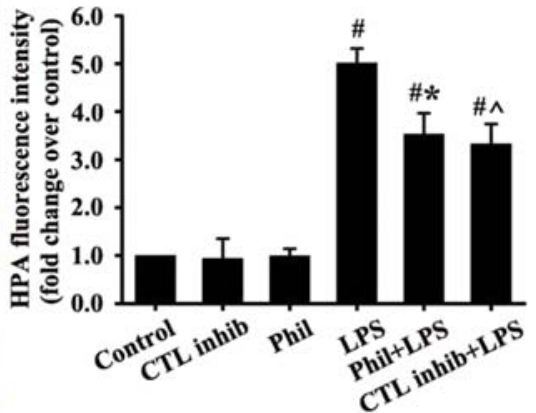

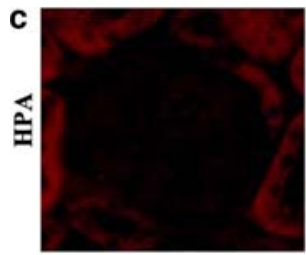
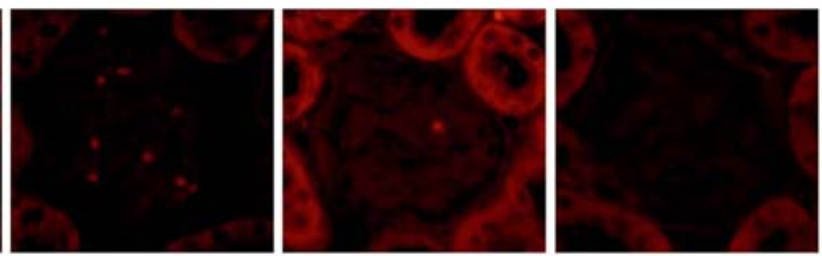

d
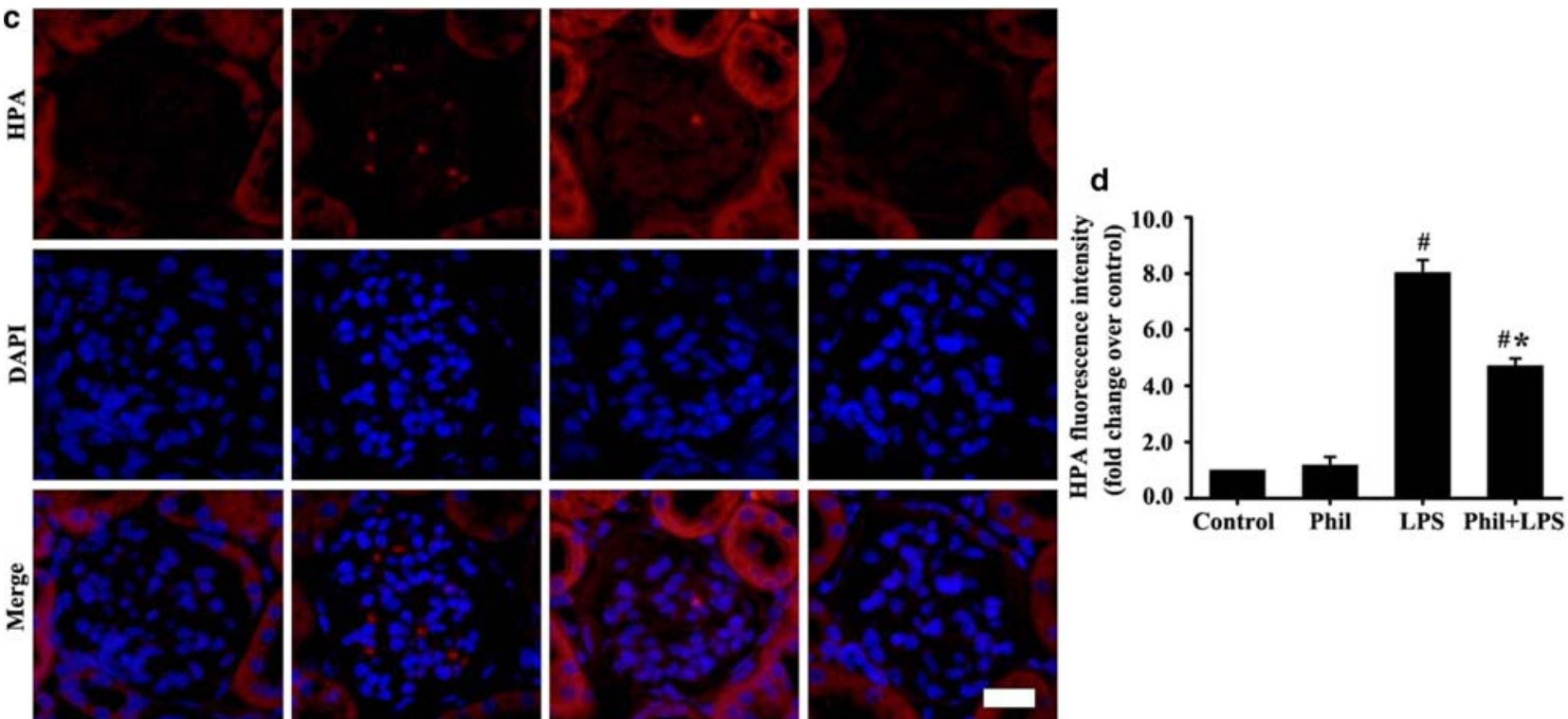

LPS

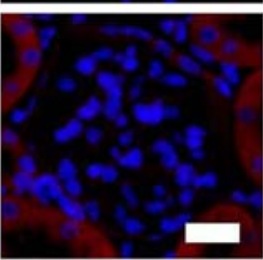

Phil+LPS

Fig. 6. Effects of Phil on HPA in LPS-stimulated EA.hy926 cells and mice. HPA contents a in vitro (magnification, $\times 200$; scale bar, $50 \mu \mathrm{m})$ and $\mathbf{c}$ in vivo (magnification, $\times 400$; scale bar, $20 \mu \mathrm{m}$ ). b, d Fluorescence intensity analysis of a and $\mathbf{c}$, respectively. All data are presented as the mean \pm SD of three independent experiments ( $n=8$ in each group). ${ }^{\#} P<0.05$ compared with the control group; ${ }^{*} P<0.05$ compared with the LPS group; ${ }^{\#} \wedge P<0.05$ compared with the LPS group.

\section{DISCUSSION}

Epithelial cells, basal membranes, and podocytes constitute the glomerular selective filtration membranes. Basement membranes and podocytes play important roles in glomerular filtration membranes [22]. The glycocalyx is essential for glomerular filtration [23, 24] and composed of HS, chondroitin sulfate, hyaluronic acid, and syndecan, among which HS is the most abundant. The glycocalyx coats the insides of the vessel lumen and is closely related to physical barriers and information transmission. An incomplete glycocalyx structure can lead to insufficiency of glomerular filtration and urinary albumin [23]. Therefore, protecting the endothelial glycocalyx against damage can help ameliorate kidney injury. In this study, the results confirmed the mechanism of damage of the endothelial glycocalyx HS in EA.hy926 cells and mice and showed that Phil significantly improves this damage.

Enzyme degradation and ROS accumulation are significant factors of HS shedding [9]. While cathepsin L is 

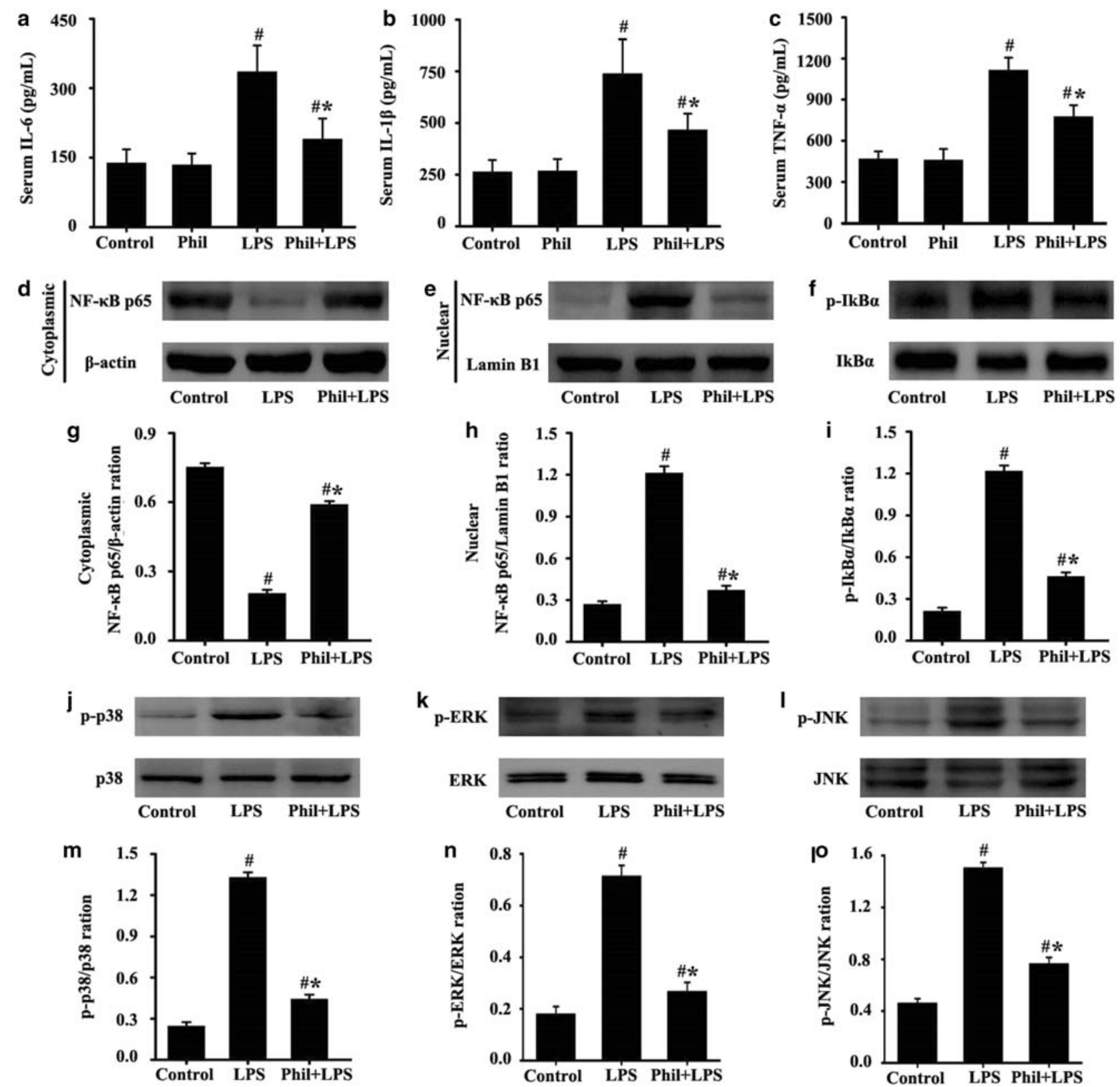

Fig. 7. Effects of Phil on inflammatory injury in LPS-induced AKI mice. Serum a IL-6, $\mathbf{b}$ IL-1 $\beta$, and $\mathbf{c}$ TNF- $\alpha$ levels were measured by the corresponding ELISA kits. The NF-KB and MAPK inflammatory pathways in kidneys were detected by Western blot. $\beta$-actin or Lamin B1 was used as the internal control. $\mathbf{g}, \mathbf{h}, \mathbf{i}, \mathbf{m}, \mathbf{n}, \mathbf{o}$ Relative intensity analysis of $(\mathbf{d}),(\mathbf{e}),(\mathbf{f}),(\mathbf{j}),(\mathbf{k})$, and (l), respectively. All data are presented as the mean \pm SD of three independent experiments ( $n=8$ in each group). ${ }^{\#} P<0.05$ compared with the control group; ${ }^{\# *} P<0.05$ compared with the LPS group.

known to regulate dengue virus NS1-induced endothelial glycocalyx damage [8], the mechanisms of endothelial glycocalyx damage have not been studied in EA.hy926 cells and LPS-induced AKI mice. In the present study, addition of cathepsin L inhibitor inhibited cathepsin L and HPA expression levels and protected against glycocalyx HS shedding in EA.hy926 cells (Figs. 5 and 6). Addition of NAC decreased the ROS production induced by LPS in EA.hy926 cells and alleviated damage to the glycocalyx HS to some extent (Fig. 4). Therefore, inhibition of cathepsin L or ROS 
production could protect against glycocalyx HS damage in EA.hy926 cells. Pretreatment with Phil can significantly improve glycocalyx damage by inhibiting cathepsin L or ROS production in EA.hy926 cells and mice.

AKI is accompanied by severe inflammatory reactions that can lead to kidney damage and declines in renal function [25]. The NF-KB and MAPK pathways play an important role in mediating the inflammatory response. As the upstream signals of the inflammatory response, NF- $\mathrm{KB}$ and MAPK are activated to induce downstream inflammatory responses, which produce excess inflammatory cytokines, such as IL-6, TNF- $\alpha$, and IL- $1 \beta$. AKI can initiate the inflammatory cascade associated with renal ischemia/ reperfusion injury and aggravate the development of renal injury [26]. The inflammatory response has a close relationship with the endothelial glycocalyx; indeed, this response can damage the endothelial glycocalyx and accelerate the spread of inflammation $[27,28]$. The results of the present study showed that pretreatment with Phil can significantly inhibit inflammatory responses in LPS-induced AKI mice, thereby reducing renal damage and improving the recovery of renal function.

The pathogenic factors of AKI are complex. This study analyzed the mechanisms of Phil in LPS-induced AKI mice. Further work must be conducted to determine whether Phil can protect AKI induced by other factors, such as trauma, nephrotoxic drugs, and ischemia/reperfusion injury [29-31].

Complete analysis of the pharmacokinetic changes in mice will provide insights into the distribution of Phil in different mice organs. However, because of limitations related to the laboratory conditions, this study did not analyze pharmacokinetic changes in mice. Nevertheless, this study may provide a new theoretical basis for the development of new therapeutic drugs for AKI.

\section{ACKNOWLEDGMENTS}

This work was supported by funding from the Natural Science Foundation of Shandong Province, China (ZR2017MH065), Young Teachers' Training and Funding Project of Binzhou Medical University, National Natural Science Foundation of China (No: 81670078).

\section{REFERENCES}

1. Sigurjonsdottir, V.K., S. Chaturvedi, C. Mammen, and S.M. Sutherland. 2018. Pediatric acute kidney injury and the subsequent risk for chronic kidneydisease: Is there cause for alarm? Pediatric Nephrology 33 (11): 2047-2055.

2. Aujla, H., T. Kumar, M. Woźniak, W. Dott, N. Sullo, L. Joel-David, T. Morris, C. Brookes, S. Barber, and G.J. Murphy. 2018. Effect of sildenafil (revatio) on postcardiac surgery acute kidney injury: a randomised, placebo-controlled clinical trial: the REVAKI-2 trial protocol. Open Heart 5 (2): e000838.

3. Jin, Y., X. Shao, B. Sun, C. Miao, Z. Li, and Y. Shi. 2017. Urinary kidney injury molecule-1 as an early diagnostic biomarker of obstructive acute kidney injury and development of a rapid detection method. Molecular Medicine Reports 15 (3): 1229-1235.

4. Romagnoli, S., Z. Ricci, and C. Ronco. 2018. CRRT for sepsisinduced acute kidney injury. Current Opinion in Critical Care 24 (6): 483-492.

5. Senouthai, S., J. Wang, D. Fu, and Y. You. 2019. Fractalkine is involved in lipopolysaccharide-induced podocyte injury through the $\mathrm{Wnt} / \beta$-catenin pathway in an acute kidney injury mouse model. Inflammation 42 (4): 1287-1300.

6. Mir, S.M., H.G. Ravuri, R.K. Pradhan, S. Narra, J.M. Kumar, M. Kuncha, S. Kanjilal, and R. Sistla. 2018. Ferulic acid protects lipopolysaccharide-induced acute kidney injury by suppressing inflammatory events and upregulating antioxidant defenses in Balb/c mice. Biomedicine \& Pharmacotherapy 100: 304-315.

7. Garsen, M., A.L. Rops, T.J. Rabelink, J.H. Berden, and J. van der Vlag. 2014. The role of heparanase and the endothelial glycocalyx in the development of proteinuria. Nephrology Dialysis Transplantation 29 (1): 49-55.

8. Puerta-Guardo, H., D.R. Glasner, and E. Harris. Dengue virus NS1 disrupts the endothelial glycocalyx, leading to hyperpermeability. PLoS Pathogens 12 (7): e1005738.

9. Singh, A., R.D. Ramnath, R.R. Foster, E.C. Wylie, V. Fridén, I. Dasgupta, B. Haraldsson, G.I. Welsh, P.W. Mathieson, and S.C. Satchell. 2013. Reactive oxygen species modulate the barrier function of the human glomerular endothelial glycocalyx. PLoS One 8 (2): e55852.

10. Shen, W.C., C.J. Liang, T.M. Huang, C.W. Liu, S.H. Wang, G.H. Young, J.S. Tsai, Y.C. Tseng, Y.S. Peng, V.C. Wu, and Y.L. Chen. 2016. Indoxyl sulfate enhances IL-1 $\beta$-induced E-selectin expression in endothelial cells in acute kidney injury by the ROS/MAPKs/ NFKB/AP-1 pathway. Archives of Toxicology 90 (11): 2779-2792.

11. Yang, L., X. Zhou, W. Huang, Q. Fang, J. Hu, L. Yu, N. Ma, and W. Zhang. 2017. Protective effect of phillyrin on lethal LPS-induced neutrophil inflammationin zebrafish. Cellular Physiology and Biochemistry 43 (5): 2074-2087.

12. Zhong, W.T., Y.C. Wu, X.X. Xie, X. Zhou, M.M. Wei, L.W. Soromou, X.X. Ci, and D.C. Wang. 2013. Phillyrin attenuates LPS-induced pulmonary inflammation via suppression of MAPK and NF-KB activation in acute lung injury mice. Fitoterapia 90 : $132-139$.

13. Wei, T., W. Tian, H. Yan, G. Shao, and G. Xie. 2014. Protective effects of phillyrin on $\mathrm{H}_{2} \mathrm{O}_{2}$-induced oxidative stress and apoptosis in PC12 cells. Cellular and Molecular Neurobiology 34 (8): 11651173.

14. Zhang, W.L., L. Zhu, and J.G. Jiang. 2014. Active ingredients from natural botanicals in the treatment of obesity. Obesity Reviews 15 (12): 957-967.

15. Qu, X.Y., Q.J. Li, H.M. Zhang, X.J. Zhang, P.H. Shi, X.J. Zhang, J. Yang, Z. Zhou, and S.Q. Wang. 2016. Protective effects of phillyrin against influenza A virus in vivo. Archives of Pharmacal Research 39 (7): 998-1005.

16. Schröder, S., S. Broese, J. Baake, D. Juerß, S. Kriesen, G. Hildebrandt, and K. Manda. 2019. Effect of ionizing radiation on human EA.hy926 endothelial cells under inflammatory conditions 
and their interactions with A549 tumour cells. Journal of Immunology Research 2019: 9645481.

17. Cheng, Y., X. Liang, L. Feng, D. Liu, M. Qin, S. Liu, G. Liu, and M. Dong. 2017. Effects of phillyrin and forsythoside A on rat cytochrome P450 activities in vivo and in vitro. Xenobiotica 47 (4): 297303.

18. Yu, C., D. Qi, J.F. Sun, P. Li, and H.Y. Fan. 2015. Rhein prevents endotoxin-induced acute kidney injury by inhibiting NF- $\mathrm{kB}$ activities. Scientific Reports 5: 11822.

19. Zhang, Q., X. Gong, G. Kuang, R. Jiang, T. Xie, H. Tie, X. Chen, K. Li, J. Wan, and B. Wang. 2018. Ferulic acid protected from kidney ischemia reperfusion injury in mice: possible mechanism through increasing adenosine generation via HIF-1 $\alpha$. Inflammation 41 (6): 2068-2078.

20. Zhang, L., Y. Gu, H. Li, H. Cao, B. Liu, H. Zhang, and F. Shao. 2018. Daphnetin protects against cisplatin-induced nephrotoxicity by inhibiting inflammatory and oxidative response. International Immunopharmacology 65: 402-407.

21. Ma, J., Y.T. Li, S.X. Zhang, S.Z. Fu, and X.Z. Ye. 2019. MiR-590-3p attenuates acute kidney injury by inhibiting tumor necrosis factor receptor-associated factor 6 in septic mice. Inflammation 42 (2): 637-649.

22. Lahdenkari, A.T., K. Lounatmaa, J. Patrakka, C. Holmberg, J. Wartiovaara, M. Kestilä, O. Koskimies, and H. Jalanko. 2004. Podocytes are firmly attached to glomerular basement membrane in kidneys with heavy proteinuria. Journal of the American Society of Nephrology 15 (10): 2611-2618.

23. Garsen, M., O. Lenoir, A.L. Rops, H.B. Dijkman, B. Willemsen, T.H. van Kuppevelt, T.J. Rabelink, J.H. Berden, P.L. Tharaux, and J. van der Vlag. 2016. Endothelin-1 induces proteinuria by heparanase-mediated disruption of the glomerular glycocalyx. Journal of the American Society of Nephrology 27 (12): 3545-3551.
24. Fridén, V., E. Oveland, O. Tenstad, K. Ebefors, J. Nyström, U.A. Nilsson, and B. Haraldsson. 2011. The glomerular endothelial cell coat is essential for glomerular filtration. Kidney International 79 (12): 1322-1330.

25. Skube, S.J., S.A. Katz, J.G. Chipman, and C.J. Tignanelli. 2018. Acute kidney injury and sepsis. Surgical Infections (Larchmt) 19 (2): 216-224.

26. Fontana, J., A. Vogt, A. Hohenstein, U. Vettermann, E. Doroshenko, E. Lammer, B.A. Yard, and S. Hoeger. 2017. Impact of steroids on the inflammatory response after ischemic acute kidney injury in rats. Indian Journal of Nephrology 27 (5): 365-371.

27. Lipowsky, H.H. 2018. Role of the glycocalyx as a barrier to leukocyte-endothelium adhesion. Adv. Exp. Med. Biol 1097: 51-68.

28. Rops, A.L., M.A. Loeven, J.J. van Gemst, I. Eversen, X.M. Van Wijk, H.B. Dijkman, T.H. van Kuppevelt, J.H. Berden, T.J. Rabelink, J.D. Esko, and J. van der Vlag. 2014. Modulation of heparan sulfate in the glomerular endothelial glycocalyx decreases leukocyte influx during experimental glomerulonephritis. Kidney International 86 (5): 932-942.

29. Perkins, Z.B., G. Captur, R. Bird, L. Gleeson, B. Singer, and B. O'Brien. 2019. Trauma induced acute kidney injury. PLoS One 14 (1): e 0211001.

30. González-Guerrero, C., J.L. Morgado-Pascual, P. Cannata-Ortiz, M.A. Ramos-Barron, C. Gómez-Alamillo, M. Arias, S. Mezzano, J. Egido, M. Ruiz-Ortega, A. Ortiz, and A.M. Ramos. 2018. CCL20 blockade increases the severity of nephrotoxic folic acid-induced acute kidney injury. Journal of Pathology 246 (2): 191-204.

31. Li, J.H., Y. Tang, J. Lv, X.H. Wang, H. Yang, P.M.K. Tang, X.R. Huang, Z.J. He, Z.J. Zhou, Q.Y. Huang, J. Klug, A. Meinhardt, G. Fingerle-Rowson, A.P. Xu, Z.H. Zhen, and H.Y. Lan. 2019. Macrophage migration inhibitory factor promotes renal injury induced by ischemic reperfusion. Journal of Cellular and Molecular Medicine 23 (6): 3867-3877. 